\title{
Design of Wideband Vibration-based Electromagnetic Generator by means of Dual-resonator
}

\author{
B.L. Ooi ${ }^{\mathrm{a}, *}$, A. Rashid. A. Aziz ${ }^{\mathrm{a}}$, J.M. Gilbert ${ }^{\mathrm{b}}$ \\ ${ }^{a}$ Centre for Automotive Research and Electric Mobility, Universiti Teknologi PETRONAS (UTP), Bandar Seri Iskandar, 31750 Tronoh, Perak, Malaysia \\ ${ }^{\mathrm{b}}$ School of Engineering, University of Hull, Hull, HU6 7RX, United Kingdom
}

\begin{abstract}
This paper describes the design of a wideband electromagnetic energy harvester that utilizes a novel dual-resonator method to improve the operational frequency range of the vibration-based generator. The device consists of two separate resonator systems (coil \& magnet), which each comply with their respective resonance frequencies. This is because both resonator are designed in such a way that both magnet and coil components will oscillate at an additive phase angle, and hence create greater relative motion between the two dominating resonance frequencies, which realizes the wideband generator. Each resonator system consists of a distinctive cantilever beam, one attached with four magnets and steel keepers, the other attached with a copper coil and stainless steel holder as the free end mass. Both cantilevers are clamped and fitted to a common base that is subjected to a vibration source. Basic analytical models are derived and a numerical model is implemented in MATLAB-Simulink. Electromagnetic, structural modal and static mechanical analysis for the design of the prototype are completed using ANSYS finite element tools. For a $0.8 \mathrm{~ms}^{-2}$ acceleration, the open-loop voltage obtained from the experiment system show a good correlation with those from the simulation. Peak induced voltage is measured to be $259.5 \mathrm{~V}_{\text {rms }}$ as compared to $240.9 \mathrm{~V}_{\text {rms }}$ from the simulator at $21.3 \mathrm{~Hz}$, which implies an error range of $7.7 \%$. The results also indicate that there is a maximum of $58.22 \%$ improvement in the induced voltage within the Intermediate Region which occurs at the intersection point between the output response plots of two single resonator generators.
\end{abstract}

Keywords: Energy harvesting, Wideband harvester, Dual-resonator, Energy scavenging, Electromagnetic generator

\section{Introduction}

Over recent years, by using the latest advances in technology, miniature sensor systems can be manufactured and integrated with low-power wireless technology and operates at powers as low as $100 \mu \mathrm{W}$ [1]. Wireless Sensor Network (WSN) systems can be established by combining these miniature sensors together to form an attractive monitoring system that can be widely used for applications in automotive [2], environmental monitoring [3], health care [4], logistic \& retails [5] and military industries [6]. At present, most of these systems are still powered by batteries which not only limit the sensor miniaturization process but also provide finite energy capacity and can create environmental pollution caused by the disposal of used batteries. Hence, in the past few years, alternative power sources have become highly desirable and have attracted great interests from many academic research clusters, including applied science, mechanical and electrical engineering.

One of the promising alternative solutions to a battery is by energy scavenging from ambient sources such as solar, thermal, wind and vibration. These ambient energy resources are redundant and have almost unlimited lifetime. Some of these energy sources have some limitations like in the case of wind energy as it cannot be used in locations where there is a weak wind density. In the case of solar energy, it will be less effective in location where there is absence of sunlight or dim light is present. Among these energy sources, the vibration sources claimed to be the most attractive due to their availability, high power density and easiness of integration with miniature sensors [7].

Vibration is a phenomenon whereby the source oscillates about an equilibrium point. Vibration, also known as kinetic energy, is very often present and abundant, typically from vehicle vibrations, human activities and noisy environments. In order to capture these motions and convert them to electrical energy, a mechanical coupling between the oscillating source and the physical device capable of generating electricity is required. In this context, many linear vibration-to-electricity conversion mechanisms have been presented in recent years [8]. They are piezoelectric [9], electrostatic [10] and electromagnetic [11]. Although there are many types of conversion mechanisms, no matter which principle is exploited, all of these aforementioned conversion harvesters have the same problem, in which the vibration-based energy harvester device will provide the optimal power when their resonance frequency matches the ambient excitation frequency. If the excitation frequency deviates from the

\footnotetext{
* Corresponding author. +60 124366628

Email addresses: benglee_85@hotmail.com, benglee.ooi@ petronas.com.my
} 
resonance frequency of the oscillator, the harvester performance will dramatically drop. However, in the majority of practical cases, the frequencies present in a particular location may not be well known or may vary over a certain range of spectrum. For instance, the dominant vibration frequency on an automotive engine changes when the gear ratio of the transmission is shifted, so it would clearly be advantageous to have a single design that operates effectively over a range of the vibration spectrum. Therefore how to broaden the operational bandwidth of a harvester has become one of the most challenging issues.

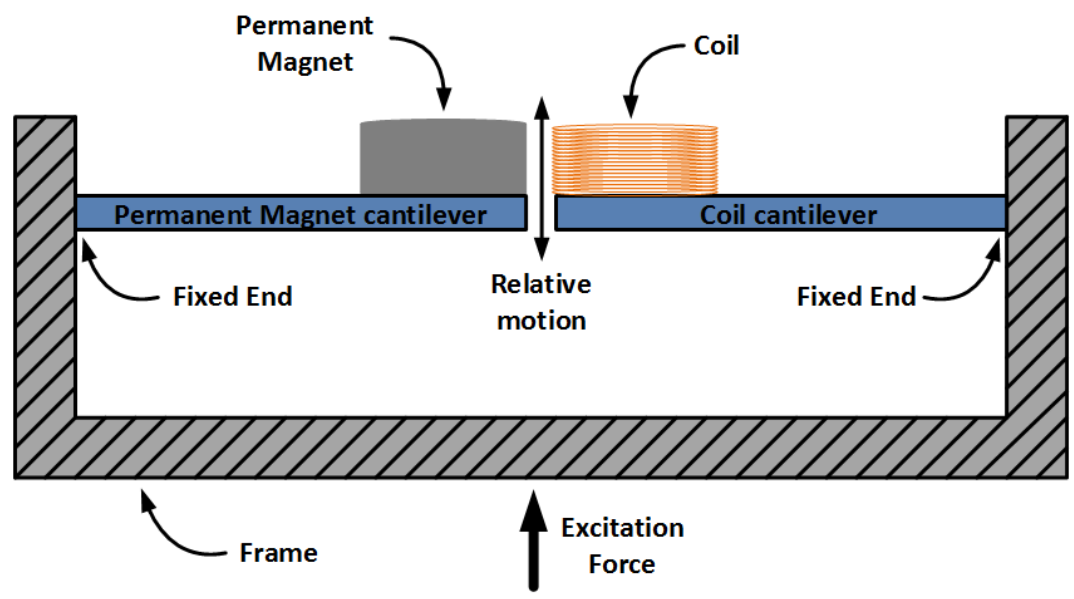

Fig. 1-Dual-resonator electromagnetic generator

In order to resolve the frequency matching issue, several adaptive harvester solutions have been proposed in the literature. They are categorized into 4 approaches: resonance frequency tuning [12][13], non-linear oscillation [1][14], frequency-upconversion (FupC) [15]-[16] and multiple harvesters [17][18]. However, every aforementioned approach has its own advantages and drawbacks in term of generator size, output power density, design complexity and so on. Although there are many proposed solutions available, until now a dual-resonator method has not been considered. In this paper, a novel method to widen the generator bandwidth is presented (Fig. 1). The method proposes is to implement an energy harvester which involves two independent resonating components (coil and permanent magnet sets), having dissimilar resonance frequencies, fitted to a common inertia frame and generating energy through their relative motion during the existence of the external excitation force. Due to the additive phase angle between the two resonators during the oscillation, higher induced output can be achieved in the Intermediate Region between the resonance frequencies of the two cantilever systems (as will be shown in Section 4.2). In this paper, a basic analytical model of the dual-resonator generator is presented and the proposed model is then numerically implemented in the MATLAB-Simulink solver. The design of the prototype is described; the magnetic field distribution, cantilever resonance and stress are estimated based on ANSYS finite element modeling (FEM) tools. Finally, the induced output voltage from the generator is recorded. The obtained output is discussed by comparing its simulations with experimental results.

\section{Basic Theory}

A common single resonator inertia vibration-based generator device can generally be modelled as a second-order mass, spring and damper systems with external base excitation as illustrated in Fig. 2, which is well documented by William and Yates [19]. The system consists of a seismic mass, $m$, a spring of stiffness, $k$, the damping coefficient, $b$, the time varying position, $z(t)$, of the seismic mass relative to the support casing that is driven by $\mathrm{y}(\mathrm{t})$, the time varying external excitation force. The motion equation of this system may be written as

$m \ddot{z}(t)+b \dot{z}(t)+k z(t)=-m \ddot{y}(t)$ 


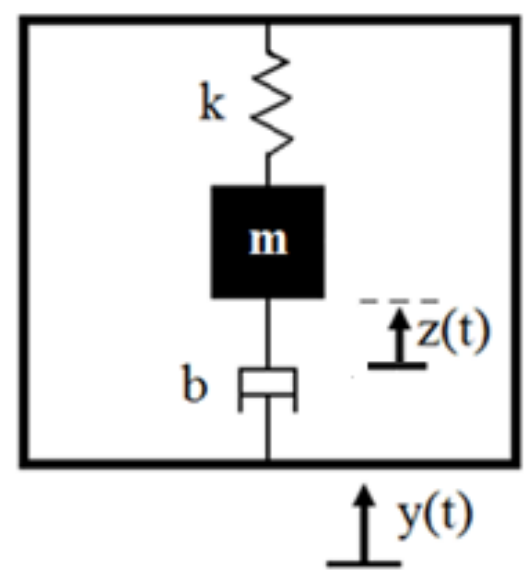

Fig. 2- Model of common inertial generator

Assuming that the vibrating source of the device relative to the inertial frame is in sinusoidal form and can be described by $y(t)=\mathrm{Y} \sin (\omega t)$, then the particular solution for $z(t)$ is

$z(t)=\left[\frac{Y \omega^{2}}{\sqrt{\left(\omega_{o}^{2}-\omega^{2}\right)^{2}+\left(2 \omega_{o} \omega \zeta_{T}\right)^{2}}}\right] \sin (\omega t-\psi)$

and the phase angle $\psi$ is

$\psi=\tan ^{-1}\left(\frac{2 \omega_{o} \omega \zeta_{T}}{\omega_{o}^{2}-\omega^{2}}\right)$

where $\omega_{o}=\sqrt{\mathrm{k} / \mathrm{m}}$ is the natural frequency of the resonant system and $\zeta_{T}=b /\left(2 m \omega_{o}\right)$ is the damping factor. The relationship between phase angle and normalized frequency $\left(r=\omega / \omega_{o}\right)$ given in (3) is plotted in Fig. 3 for a range of value of $\zeta_{T}$. It can be seen that the $90^{\circ}$ phase different occurs when the excitation vibration frequency matches the resonance frequency $\left(\omega=\omega_{o}\right.$ or $r=$ 1). It may also be noted that increasing the damping, $\zeta_{T}$, has the effect of changing the behavior of the phase angle when the excitation frequency is swept from lower to higher frequency than the resonance. For a lower value of damping, e.g. $\zeta_{T}=0.01$, the phase $\psi$ will remains almost $0^{\circ}$ and starts to change significantly only when the sweeping frequency is nearly matched to the resonance frequency. Then, the phase will stays at nearly $180^{\circ}$ after the excitation frequency is swept above resonance. For higher values of $\zeta_{T}=0.05$ and 0.1 , the phase starts to shift more slowly during the beginning stage of the sweeping but requires larger frequency deviation above resonance to reach the $180^{\circ}$ mark. Thus, this implies that for a given mass, spring and damper system, a resonator phase angle will remain at a value close to $0^{\circ}$ when the excitation frequency is lower than the resonance, $90^{\circ}$ during the resonance and at a value close to $180^{\circ}$ when the frequency is higher than the resonance.

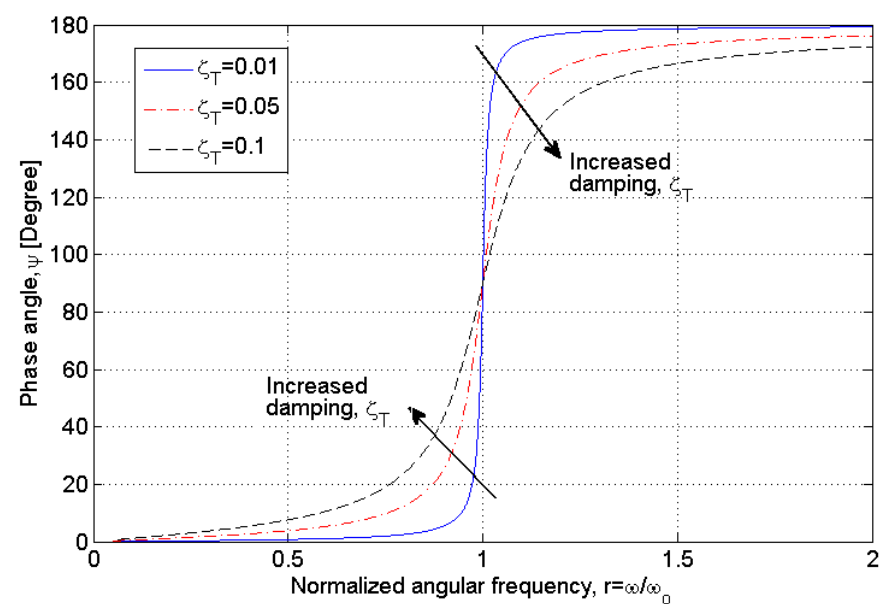

Fig. 3-Relationship between phase angle and normalize frequency 
Unlike the previous literature work, the dual-resonator generator has both the coil and the magnet excited and allowed to oscillate together under the common vibrating source. Due to the dissimilarity of the resonances, the system should exhibit two resonance frequencies; one from the coil resonating component, another from the permanent magnet resonating component. Hence, this generator can be modelled as in Fig. 4. Where $x(t)$ is the position of the seismic mass relative to its equilibrium position, the subscripts $m$ and $c$ denote the magnet and coil components, respectively. The damper $b_{r}$ represents the sum of the damping caused by the electromagnetic coupling and air resistance between magnet and coil, The $\mathrm{b}_{\mathrm{r}}$ losses is assumed to be relatively small comared to the damping from losses in the cantilever material (as justified by later results in Section 4.2), hence it will not be considered in the following analytical and numerical modelling.

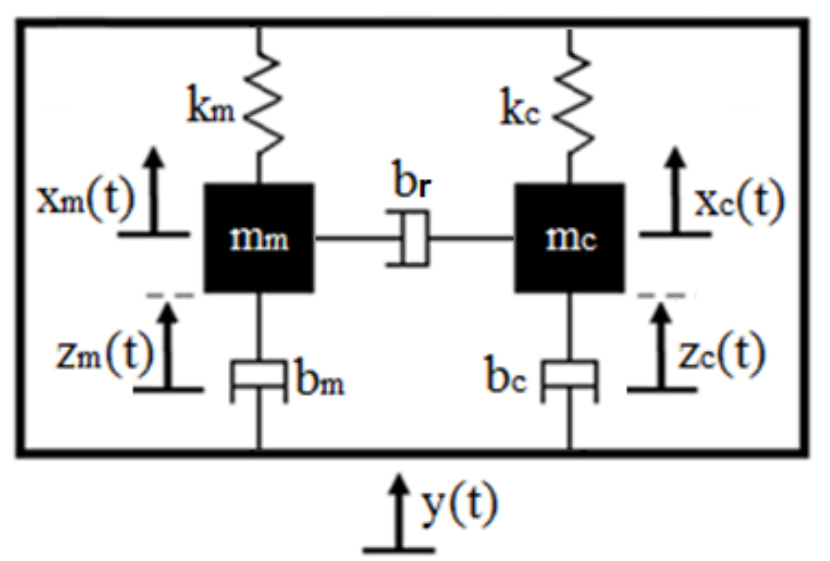

Fig. 4-Model of dual-resonator generator

The relative motion can be determined by subtracting the coil's motion from the magnet's motion or vice versa. In this paper, the latter is considered and therefore the relative motion between the two seismic masses can expressed as

$x_{r}(t)=x_{c}(t)-x_{m}(t)$

Given that $z_{c}(t)=x_{c}(t)-y(t)$ and $z_{m}(t)=x_{m}(t)-y(t)$, hence (4) can be written as $x_{r}(t)=z_{r}(t)$ and upon differentiation lead to

$\dot{x}_{r}(t)=\dot{z}_{r}(t)$

Hence, it is possible to use $z_{r}(t)$ instead of $x_{r}(t)$ in the calculation for the seismic masses relative motion. In this paper, since no external electrical load will be considered, when damping is reported, it will be assumed that it is purely a mechanical damping. Referring to (2) and (3), the solutions for $\dot{z}_{c}(t), \dot{z}_{m}(t), \psi_{c}$ and $\psi_{m}$ can be given as

$$
\begin{aligned}
& \dot{z}_{c}(t)=\left[\frac{Y \omega^{3}}{\sqrt{\left(\omega_{c}^{2}-\omega^{2}\right)^{2}+\left(2 \omega_{c} \omega \zeta_{c}\right)^{2}}}\right] \cos \left(\omega t-\psi_{c}\right) \\
& \dot{z}_{m}(t)=\left[\frac{Y \omega^{3}}{\sqrt{\left(\omega_{m}^{2}-\omega^{2}\right)^{2}+\left(2 \omega_{m} \omega \zeta_{m}\right)^{2}}}\right] \cos \left(\omega t-\psi_{m}\right) \\
& \psi_{c}=\tan ^{-1}\left(\frac{2 \omega_{c} \omega \zeta_{c}}{\omega_{c}^{2}-\omega^{2}}\right) \\
& \psi_{m}=\tan ^{-1}\left(\frac{2 \omega_{m} \omega \zeta_{m}}{\omega_{m}^{2}-\omega^{2}}\right)
\end{aligned}
$$




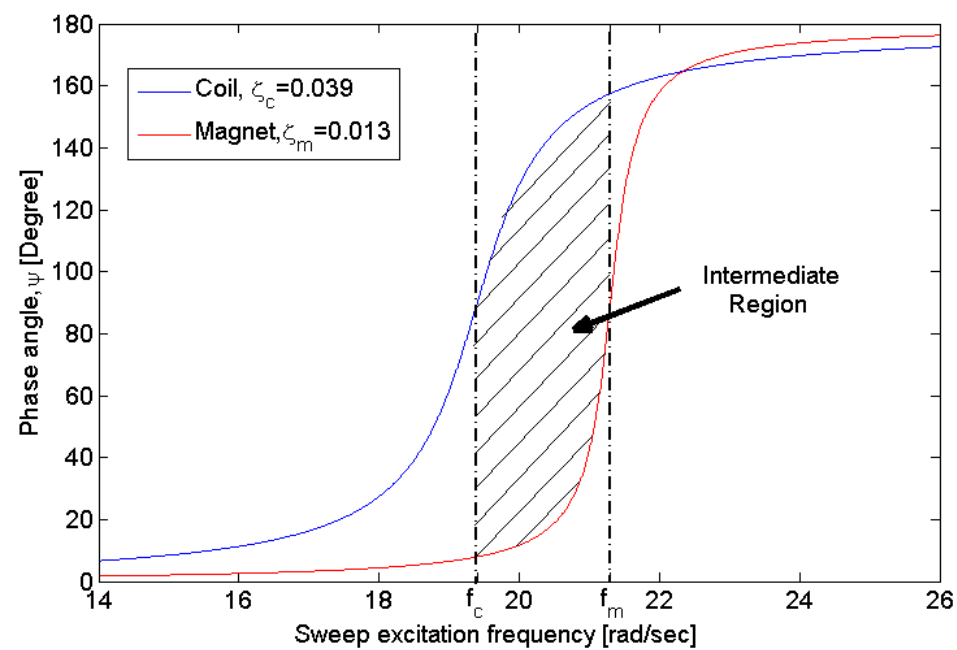

Fig. 5- Phase of coil \& magnet against the sweep frequency

The phase angle $\psi_{c}$ and $\psi_{m}$ from (8) and (9) against the sweep frequencies are plotted in Fig. 5 for resonance frequencies and damping factors for coil and magnet component of $\mathrm{f}_{\mathrm{c}}=19.4 \mathrm{~Hz}, \mathrm{f}_{\mathrm{m}}=21.3 \mathrm{~Hz}, \zeta_{c}=0.039$ and $\zeta_{m}=0.013$ (experimentally determined in Section 4.2) respectively. It may be noted that in the Intermediate Region (region between two resonance frequencies), the phase angle of coil and magnet resonator is mostly additive, meaning that the coil and magnet are mostly moving in opposite directions against each other (one moving up and one moving down). For example at $20.7 \mathrm{~Hz}$, the phase angle for coil and magnet are $149^{\circ}$ and $24.5^{\circ}$ respectively, resulting in an effective phase difference of $124.5^{\circ}$ (the perfect additive angle is $180^{\circ}$ ). Therefore, this allows the dual-resonator to improve the induced output in the Intermediate Region, hence widening the generator bandwidth.

It may be noted that apart from the phase angle, the speed of the oscillation of the cantilever beam also contributes to the effective induced output voltage of the generator. Having said that a mass, spring and damper system will only oscillate with resonance magnification when the excitation frequency coincides with the system natural frequency. Under other circumstances, the speed response of the system will be relatively small if compared to its resonance condition, especially for a low damped oscillating system [8]. Due to this, a closed form analytical solution will be complex. Therefore, a numerical solution was determined using the Simulink.

The model of the numerical solution is given as in Fig. 6, based on (1). This solution computes the relative velocity, $\dot{z}_{r}(t)$, between the coil and magnet component. The obtained velocity can be used to predict the emf induced in the pickup coil as

$V_{\text {ind }}(t)=N B l_{p} \dot{z}_{r}(t)$

where $V_{\text {ind }}(\mathrm{t})$ is the open-loop induced voltage, $\mathrm{N}$ is the number of turns for a given square coil, $1_{\mathrm{p}}$ is the coil practical side length and $\mathrm{B}$ is the magnetic field. It can be noticed that from (10), the induced voltage is proportional to the aforementioned parameters. One can assume that if all other factors remain constant once the coil and magnet are fabricated, then greater relative movement, $\dot{z}_{r}(t)$, between a magnet and a coil will generate a higher induced output voltage. 


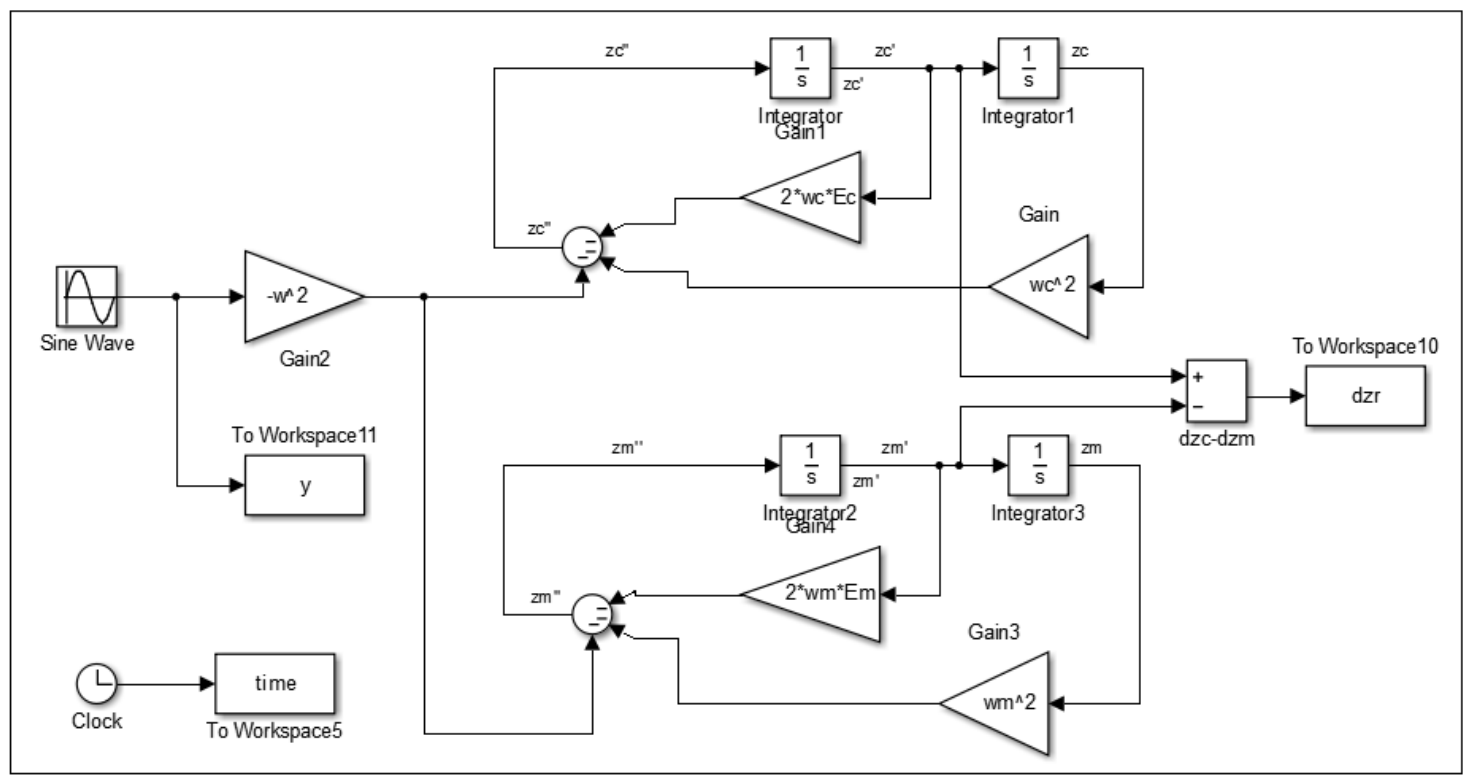

Fig. 6- Simulink model that computes the speed of both coil and magnet cantilevers

\section{Design of a Dual-resonator Generator}

\subsection{Prototype Fabrication}

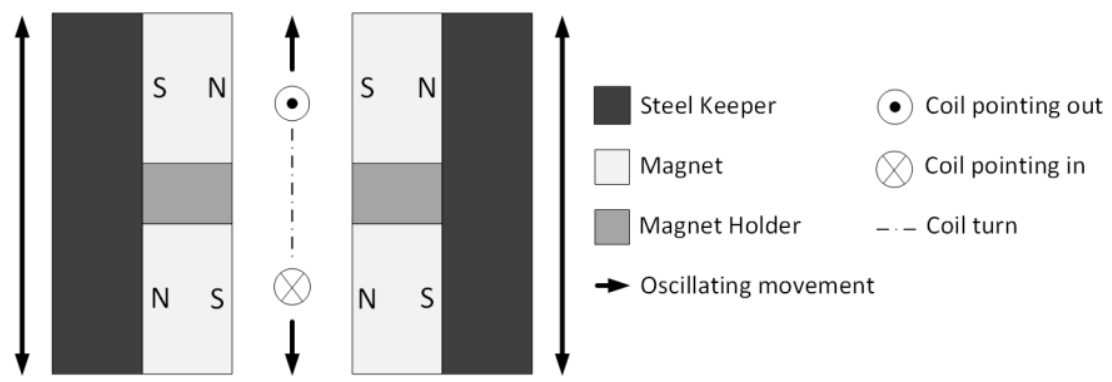

Fig. 7- Cross section view through four magnet, keeper and coil

The dual-resonator electromagnetic generator presented in this paper is based upon a cantilever structure presented in Section 1. It comprises two separate cantilever beams for magnets and coil, respectively. At the free end of these beams, the structure is designed so that a coil is sandwiched between two pairs of oppositely polarized magnets (Fig. 7). The magnetic circuit, formed from four $\mathrm{N} 35 \mathrm{NdFeB}$ magnets $(25 \times 10 \times 5 \mathrm{~mm})$, is completed by the steel keepers which couple the magnetic flux between the top and bottom magnet pairs. The magnets and soft steel keepers (AISI 1010) are assembled with a $6 \mathrm{~mm}$ air-gap in between them using a magnet holder made of Aluminum (6061). The pickup coil is held in between the gap using a coil holder fabricated from S316 stainless steel to further increase the tip mass for the coil component. Both holders are attached to two beams fabricated from a high carbon steel (AISI 1070). The beam dimension used for the coil and magnet are $16 \times 4.5 \times 0.3 \mathrm{~mm}$ and $20 \times 4.5 \times 0.7 \mathrm{~mm}$ (effective length $\times$ width $\times$ thickness), respectively. The beam dimensions required for the desired resonant frequencies were determined by finite element analysis (Section 3.3). Lastly, these beams, together with the attachment, are clamped to a common base clamper using M4 bolts. The drawing of the assembled generator is shown in Fig. 8. The second part of the drawing shows the cross section view through the center of the generator. It clearly depicts that the coil components is interleaved in between the air-gap created by the magnet pairs. It may also be noted that there are five mounting holes located at the bottom part of the base. These holes will be used to fit the fabricated generator to the vibration shaker system for data capturing. 

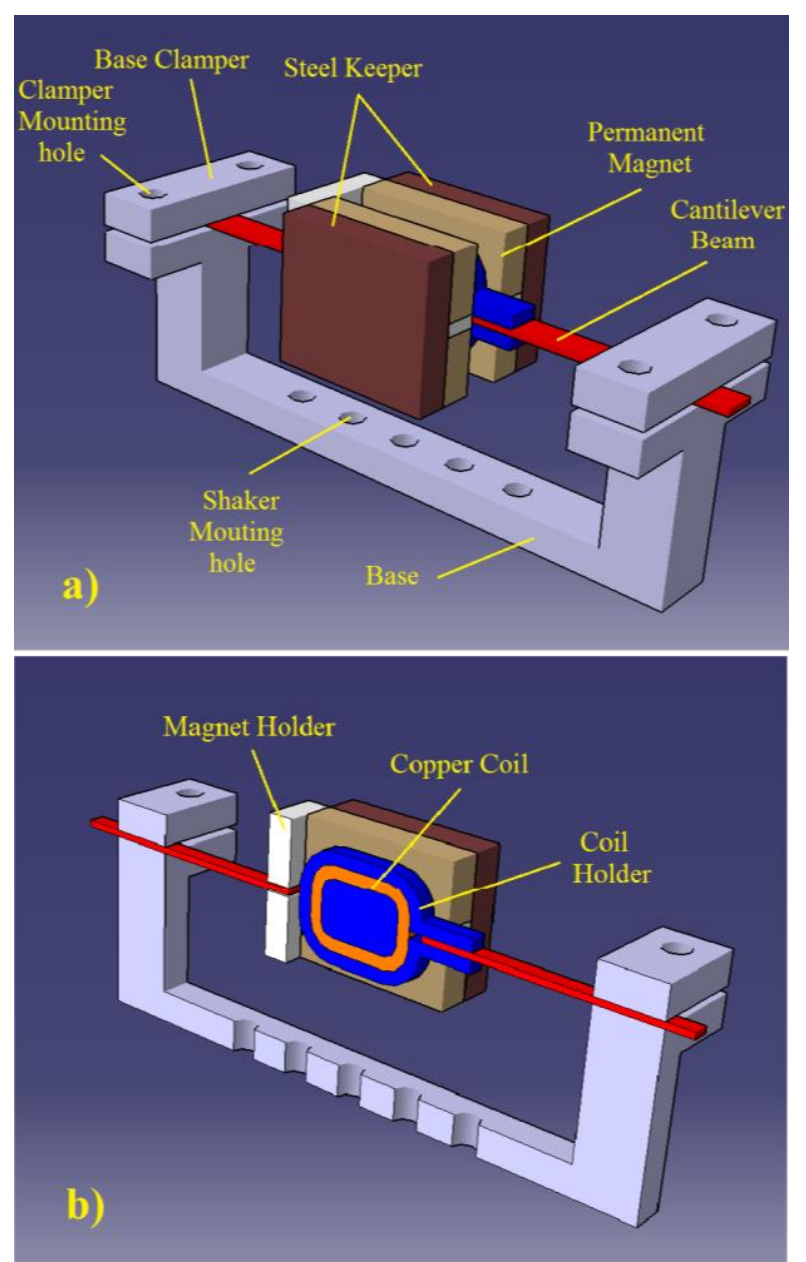

Fig. 8-a) Assembly of dual-resonator b) Cross section view of dual-resonator

\subsection{Magnetic finite element modelling}

Based on equation (10), for a greater induced voltage, one should maintain the magnetic flux density, B, in the air-gap as high as possible. The N35 magnets used in this paper have a remanence flux density $\left(\mathrm{B}_{\mathrm{r}}\right)$ and coercive force $\left(\mathrm{H}_{\mathrm{c}}\right)$ of $1.23 \mathrm{~T}$ and $900 \mathrm{kA} / \mathrm{m}$ respectively. Knowing that the energy available from a permanent magnet is fixed once it is manufactured. Therefore, one of the best way to extract the most energy from the magnet is to optimize the design of the magnetic circuit. For ease of fabrication of the coil holder, air-gap of $6 \mathrm{~mm}$ has been chosen. The wall thickness of the steel keeper is determined based on the need to carry the magnetic flux in the material without exceeding the material saturated flux density $\left(\mathrm{B}_{\mathrm{S}}\right)$ of $1.85 \mathrm{~T}$. ANSYS

Maxwell 2D magnetic finite element software was used to simulate the possible maximum magnetic flux in the material and airgap for a range of values of keeper thickness. Fig. 9 clearly illustrates that the flux concentrated in the steel keeper is greater than $\mathrm{B}_{\mathrm{S}}$ when the thickness is smaller than $5 \mathrm{~mm}$. The plot again shows that after $7 \mathrm{~mm}$, further increases the keeper thickness will not improve the flux concentration but increases generator size. Hence, optimum keeper thickness should be fixed in between 5 and $7 \mathrm{~mm}$. Fig. 10 shows the maximum possible flux density in the air-gap for a range of keeper thicknesses. This plot shows that at $5 \mathrm{~mm}$, the maximum flux density in the air-gap is $0.63 \mathrm{~T}$ and there is almost no increase in the flux density after the keeper exceeds $5 \mathrm{~mm}$ thickness. On top of that, Fig. 11 shows that the maximum flux concentration in the $5 \mathrm{~mm}$ thickness keeper is 1.784T, which is smaller than $B_{s}$. It may also be seen that there is no or negligible leakage flux outside the steel keeper. Hence, to optimize the generator size, $5 \mathrm{~mm}$ was chosen as the thickness for the steel keeper in this design. 


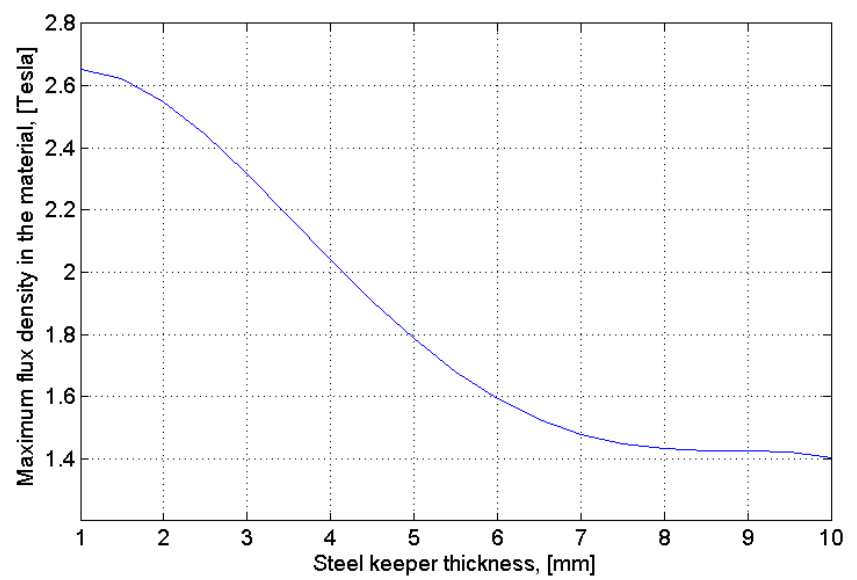

Fig. 9- Variation of flux density in the keeper for range of thickness

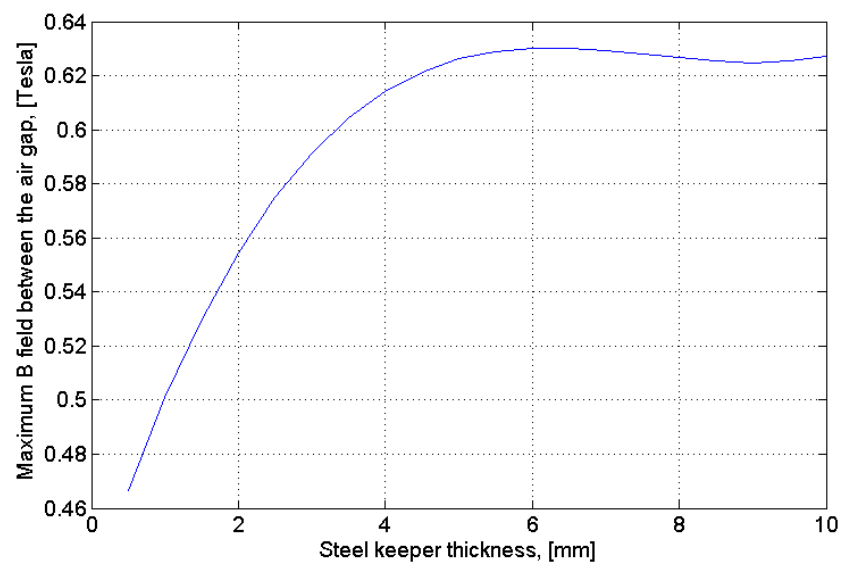

Fig. 10-Variation of flux density in the air-gap for range of thickness

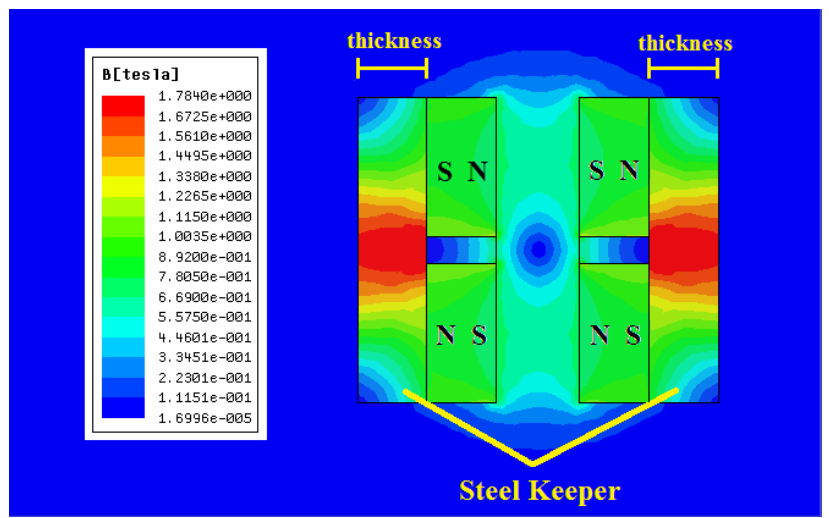

Fig. 11-Maxwell 2D simulated output for 5mm keeper thickness

If a line along the magnet's height is drawn through the center line of the air-gap in the generator, parallel to the face of the magnets then the magnetic flux density varies as shown in Fig. 12. It can be seen that the magnetic flux density is directed oppositely for the top and bottom pairs of the magnet. It is obvious that the flux density is non-linear across the region and this is mainly due to the fringing effects near to the edges. Having said that equation (10) is an approximation and only accurate when the coil is moved from a constant B field into a zero field region or vice versa. Hence, the average flux density in the air-gap may be estimated by computing the flux density along multiple paths in between the magnet and taking the average. The average magnetic field in the air-gap may be determined as $0.55 \mathrm{~T}$. However, one must realize that this estimation would deviates away from the actual value. Not to mention the effect of the coil turns in which the outer coil will has greater magnetic linkage as compare to the inner coil due to its greater effective length. 


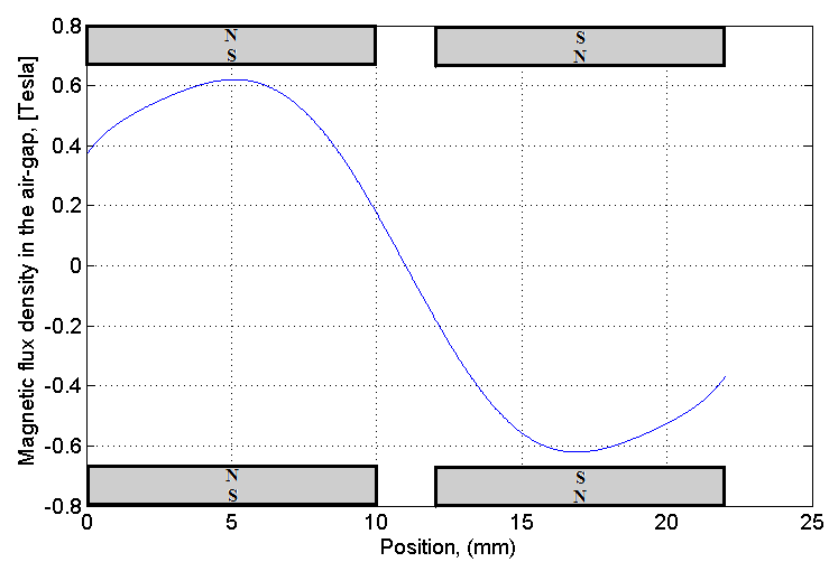

Fig. 12- Magnetic flux density in the air-gap along the magnet height

\subsection{Finite element structural analysis}

After the magnetic circuit design, the resonance frequencies for the coil and magnet beam need to be determined. For a constant amplitude acceleration force, the generator should be designed to resonate at the lowest source frequency provided that higher frequencies in the vibration spectrum do not have higher acceleration amplitude [20]. Hence, an arbitrary starting low frequency point of $20 \mathrm{~Hz}$ was selected as it has a reasonable safe margin above the minimum frequency limit of the vibration shaker system used in practical evaluations, $(5 \mathrm{~Hz})$ but no higher since this would make construction and testing more difficut. For a given amplitude of acceleration, $\mathrm{A}$, the amplitude of the displacement increases as the frequency decreases, $\mathrm{Y}=\mathrm{A} / \omega^{2}$. Hence, to maximize the displacement of the coil resonator which has a relatively low tip mass (13.04grams) as compare to the magnet resonator (85.21 grams), the former resonator was chosen to exhibit the lower resonance below $20 \mathrm{~Hz}$. The width of the cantilever beam was fixed at $4.5 \mathrm{~mm}$ for both resonators. A range of values of cantilever beam thickness and effective length were evaluated using ANSYS dynamic modal analysis in order to obtain the desired resonant frequencies. The beams $1^{\text {st }}$ and $\left(2^{\text {nd }}\right)$ vibration modes resonance frequencies were determined from finite element analysis as $\mathrm{f}_{\mathrm{c}}=18.87 \mathrm{~Hz}(347.08 \mathrm{~Hz})$ and $\mathrm{f}_{\mathrm{m}}=22.48 \mathrm{~Hz}$ $(304.84 \mathrm{~Hz})$ respectively, for the dimension as listed in Table 1. It is noted that the $2^{\text {nd }}$ and higher vibration mode frequencies for both coil and magnet beams are well above $300 \mathrm{~Hz}$. Hence it is reasonable to assume that the produced vibrations occur only in one direction. The material used for the cantilevers will affects the system damping and hence the induced output. High carbon steel was selected to be used as the beams' material because spring steel has one of the lowest damping factors, cost of material and also reasonable good material fatigue limit.

Table 1

Resonator beam dimension

\begin{tabular}{ccccc}
\hline Resonator & Material & Effective length $(\mathrm{mm})$ & Width $(\mathrm{mm})$ & Thickness $(\mathrm{mm})$ \\
\hline Coil & High carbon Steel & 16 & 4.5 & 0.3 \\
\hline Magnet & High carbon steel & 20 & 4.5 & 0.7 \\
\hline
\end{tabular}

One may argues that the resonance frequency for both coil and magnet beams can be estimated using analytical methods as given in equation (11) [21] provided that all the parameters values are known, and this would be less time consuming than the finite element modeling (FEM) method. However the former is not preferred due to the lack of accuracy. One must realize that the estimation in (11) is only sufficient to determine the resonance frequency of a cantilever system that has with a point tip mass since the equation does not consider the center of mass of the weight objects at the free end, not to mention that both of the resonator tip masses are assembled from several components. Although it is mathematically possible to determine the center of mass for each resonator, provided that the geometry dimension and weight of each component is given, this would add complexity to the calculation and the FEM method may be consider to be a more effective method.

$\omega_{o}=\sqrt{\frac{3 E I}{L^{3}\left[0.23 m_{\text {beam }}+m_{\text {tip }}\right]}}$ 
where $\mathrm{E}, \mathrm{I}, \mathrm{L} \mathrm{m}_{\text {beam }}$ and $\mathrm{m}_{\text {tip }}$ are the Young's modulus, moment of inertia, effective beam length, effective beam mass and total tip mass at the free end, respectively.

As the generator will be operated under a cyclical vibration force, to avoid fatigue to the cantilever beams, the input vibration force must not overstress and deflect the beams into permanently deformation, hence a low amplitude of $0.8 \mathrm{~ms}^{-2}$ input acceleration was selected. The maximum tip amplitude for both resonators were determined experimentally by a camera for the given input acceleration. A scale was placed behind the resonated beams, parallel to the direction of vibration while a high speed camera was placed perpendicularly, with a view of the cantilevers and scale. Thus the camera is able to continuously image the motion of the cantilevers against the scale and the vibration amplitudes may be determined from the maximum values observed on the scale. The were determined as $1.49 \mathrm{~mm}$ and $2.94 \mathrm{~mm}$ for the extremities of the coil and magnet components, respectively. Which corresponding to a displacement of $0.46 \mathrm{~mm}$ and $0.99 \mathrm{~mm}$ at the junction between the cantilever and the masses. The stress in the beam material were obtained using ANSYS structural static stress analysis tool as shown in Fig. 13. The maximum stress in the material were simulated as $104.43 \mathrm{MPa}$ and $358.26 \mathrm{MPa}$, respectively. This is well below the fatigue limits of the high carbon steel ( $495 \mathrm{MPa}$ ). Due to the linear nature of the mechanism, for the given safety margin, one may further increase the acceleration to achieved a higher output, however the increment should be scaled carefully to ensure that the stress in the material does not exceed the fatigue limits. It is also worth to mention that the cantilever dimensions (width and thickness) could be increased to accommodate larger acceleration, but this would also increase the beams' resonant frequency, which would impact the device performance.

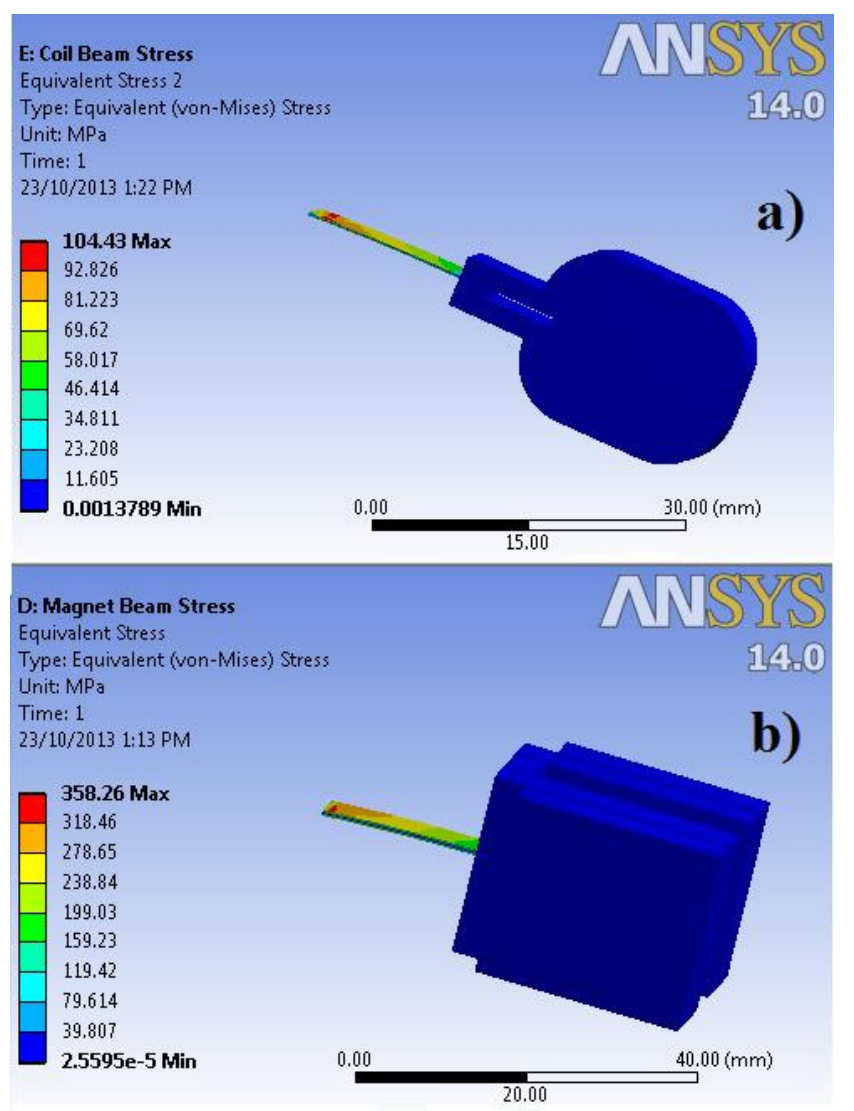

Fig. 13-ANSYS Static Structural Analysis for a) coil beam and b) magnet beam

\subsection{Coil Properties}

Since the generator is subjected to a weak vibration $\left(0.8 \mathrm{~ms}^{-2}\right)$, one can assume that the electromagnetic couple (magnet and coil) only vibrate along the vertical direction (y axis). Given that the magnetic B field is along the $\mathrm{z}$ axis, according to Faraday's Law, only the wire component along the $\mathrm{x}$ axis (perpendicular to both velocity and $\mathrm{B}$ field) will contributes in the induced voltage. Whereas the other part of the wire will contributes additional wire length to the coil, and hence to the coil's resistance, which limits the total energy available during electromagnetic generation. The relationship between the resistance, $\mathrm{R}_{\mathrm{C}}$, and total wire length of the coil, $\mathrm{L}_{\mathrm{T}}$, can be expressed as 
$R_{c}=\frac{\rho L_{T}}{A_{\text {wire }}}$

where $\rho$ is the resistivity of the conductor (copper) and $A_{\text {wire }}$ is the cross sectional area of the wire. The coil is designed in oblong shape as illustrated in Fig. 14 rather than a circular shape. The top and bottom part of the horizontal wires were carefully fitted in the air-gap (clearance $0.2 \mathrm{~mm}$ each side) to ensure the resonation is in within the upper and lower oppositely polarized magnetic pairs region, respectively, during the oscillation (Fig. 7).

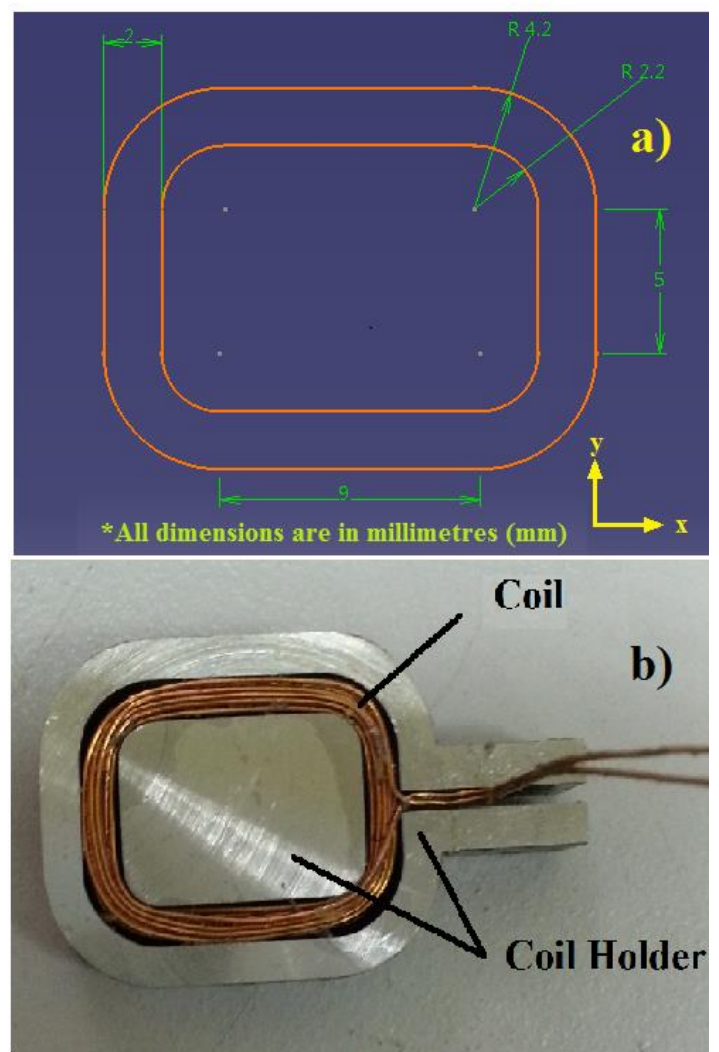

Fig. 14 - a) fabricated coil dimensions b) oblong shape coil and holder

For an oblong shape wire-wound coil, the coil fill factor, F, the ratio of volume of conductor to the volume of the practical coil, can be expressed as

$F=\frac{\pi^{2} d_{c}{ }^{4} R_{C}}{16 \rho t_{c}\left[\pi\left(R_{o}{ }^{2}-R_{i}{ }^{2}\right)+2 c_{t}\left(w_{c}+h_{c}\right)\right]}$

This gives the coil fill factor of 0.61 for the values and dimensions given in Table 2. A higher fill factor is preferable for a coil design since this implies that a higher number of wire turns are available within a given volume. A fill factor from 0.46 to 0.65 is commonly considered as a high fill winding [22]. Apart from resistive impedance, the inductance of the coil can also be considered as a function of the number of turns, cross-sectional area, and height of the coil. However, the effects of the inductance are negligible as the resistance effect is always dominant in a low frequency system. Lastly Fig. $\mathbf{1 5}$ shows the completed dual-resonator generator with some silicon glue applied to the connection edge to enhance the bonding and avoid an excessive stress to the materials. 


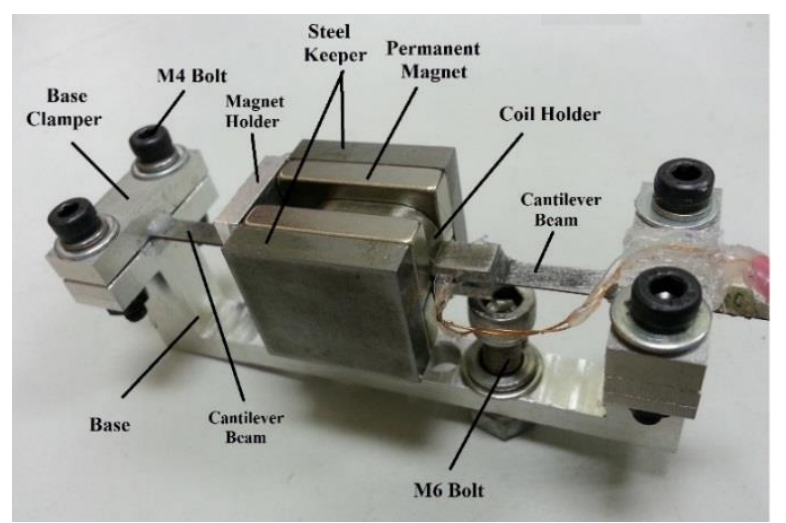

Fig. 15-Fabricated prototype of dual-resonator generator

Table 2

Description and parameter of equation (13)

\begin{tabular}{|c|c|c|c|}
\hline Description & Parameter & Value & Units \\
\hline Wire diameter & $\mathrm{d}_{\mathrm{c}}$ & 0.25 & $\mathrm{~mm}$ \\
\hline Coil resistance & $\mathrm{R}_{\mathrm{C}}$ & 2.3 & $\Omega$ \\
\hline Wire resistivity (Copper) & $\rho$ & $1.7 \times 10^{-8}$ & $\Omega \cdot \mathrm{m}$ \\
\hline Coil outer arc radius & $\mathrm{R}_{\mathrm{o}}$ & 4.2 & $\mathrm{~mm}$ \\
\hline Coil inner arc radius & $\mathrm{R}_{\mathrm{i}}$ & 2.2 & $\mathrm{~mm}$ \\
\hline Coil thickness & $\mathrm{t}_{\mathrm{c}}$ & 5.6 & $\mathrm{~mm}$ \\
\hline Coil cross section thickness & $\mathrm{c}_{\mathrm{t}}$ & 2 & $\mathrm{~mm}$ \\
\hline Coil width & $\mathrm{w}_{\mathrm{c}}$ & 9 & $\mathrm{~mm}$ \\
\hline Coil height & $\mathrm{h}_{\mathrm{c}}$ & 5 & $\mathrm{~mm}$ \\
\hline
\end{tabular}

\section{Measurements}

\subsection{Test setup}

The close-loop control system experimental setup to characterize the generator is shown in Fig. 16. The fabricated generator was mounted on top of the IMV-M060 series vibration shaker. The test profile was defined by the user using the input interface software on a personal computer and loaded to the IMV-K2 Sprint vibration controller. An accelerometer (VP-32) was mounted above the shaker to provide a feedback reference signal which allowed the controller to produce a dynamically compensated signal for the changes of the shaker's real-time vibration. The signal was then amplified by the IMV-MA1 amplifier and lastly fed to the vibration shaker unit. The generated output from the prototype was recorded and analyzed using a dynamic signal analyzer (DEWE-3020). When a sweep of frequencies are reported in this paper, it will be assumed that a vibration source of $0.8 \mathrm{~ms}^{-2}$ for a range of driving frequencies from $15 \mathrm{~Hz}$ to $24 \mathrm{~Hz}$ were used unless otherwise stated. 


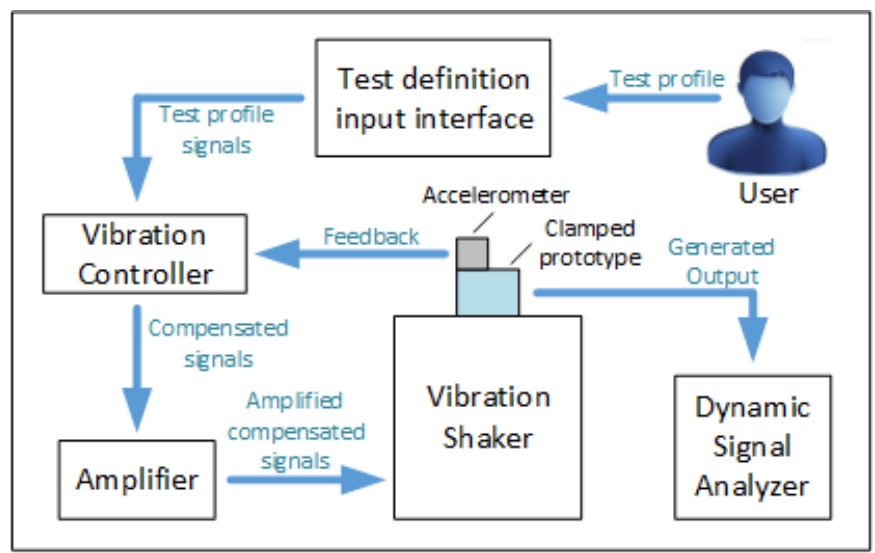

Fig. 16-Block diagram of the experimental setup

\subsection{Result and discussion}

To determine the damping factors of the coil and magnet resonators, a force impulse was applied to each resonator respectively, and the damped oscillations were recorded as shown in Fig. 17. The logarithmic decrement method was applied to identify the damping factors as

$\zeta=\frac{\ln \left(p_{1} / p_{2}\right)}{\sqrt{(2 \pi)^{2}+\left[\ln \left(p_{1} / p_{2}\right)\right]}}$

where $\mathrm{p}_{1}$ and $\mathrm{p}_{2}$ are the magnitudes of two consecutive cycle in the damped oscillation plots. Numbers of measurements were taken and the mean value of these measurements were calculated. The damping factor values were measured as 0.039 and 0.013 for coil resonator damping factor, $\zeta_{c}$, and magnet resonator damping factor, $\zeta_{m}$, respectively. The higher value of damping factor for the coil resonator is mainly due to the lighter tip mass of the coil components as compare to the magnet resonator. To further decrease this damping factor, one may replaces the existing coil holder's fabricated material with a high density non-

ferromagnetic material, such as Tungsten.

The other parameters that need to be determined are the individual resonance frequencies of the coil and magnet resonators. The coil resonance was identified by applying a vibration force with a range of sweep frequencies to the generator while holding the magnet resonator fixed with a spacer and silicon glue, then recording the frequency corresponding to the highest open- loop voltages. A similar process may be used to determine the resonance frequency of the magnet resonator but with the coil resonator fixed instead for this recording. The frequency response plot of both resonators are illustrated in Fig. 18. The peak rms open-loop voltages were experimentally measured to be $71.2 \mathrm{mV}$ at $19.4 \mathrm{~Hz}$ and $245.4 \mathrm{mV}$ at $21.3 \mathrm{~Hz}$, respectively. The values of the aforementioned damping factor and resonance frequency were then used in the modelling parameters in the MATLAB analytical and Simulink numerical solution in Fig. 5 and Fig. 6. 

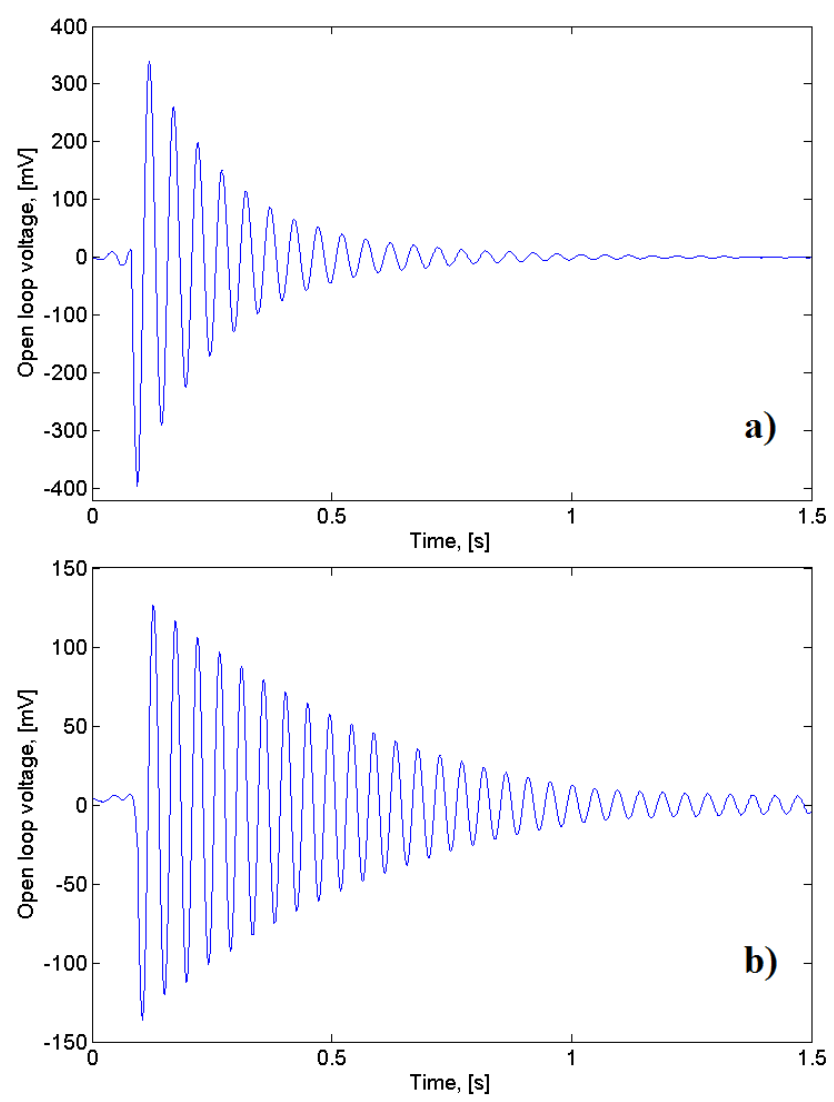

Fig. 17-Force impulse response to the a) coil resonator b) magnet resonator

The results obtained from the experimental prototype show excellent correlation with the Simulink simulation results. Fig. 19 shows that the induced rms output voltage measured experimentally from the prototype versus the simulated output from the Simulink solver when they were subjected to the aforementioned sweep frequency. It can be noticed that the experimental recorded data are generally lower than the one from the simulator. This is mainly due to the additional damping factor caused by the relative motion, measurement and setup loss during the practical experiment. At $21.3 \mathrm{~Hz}$, the open-loop voltage were determined to be $259.5 \mathrm{mV}$ and $240.9 \mathrm{mV}$ for simulation and experiment, respectively, which implies $7.7 \%$ error range. However at the lower resonance frequency (coil's resonance), the error range was determined to be $73.7 \%$ at $19.4 \mathrm{~Hz}$ for the generated voltage of $104.4 \mathrm{mV}$ and $60.1 \mathrm{mV}$. The reason for this is the higher damping factor from the coil resonator that is due to the light tip weight which causes the amplitude of the oscillation to be small. Not to mention that a higher damping factor may also degrade the effectiveness of the additive phase angle as depicted in Fig. $\mathbf{3}$ and cause the resonator to be more susceptible to the practical experimental losses. Despite these differences, the most important aspect of the behavior is that both plots look similar, indicating good agreement between the experiment and simulation. This suggests that the developed Simulink model is sufficient to characterize the dual-resonator generator device. 


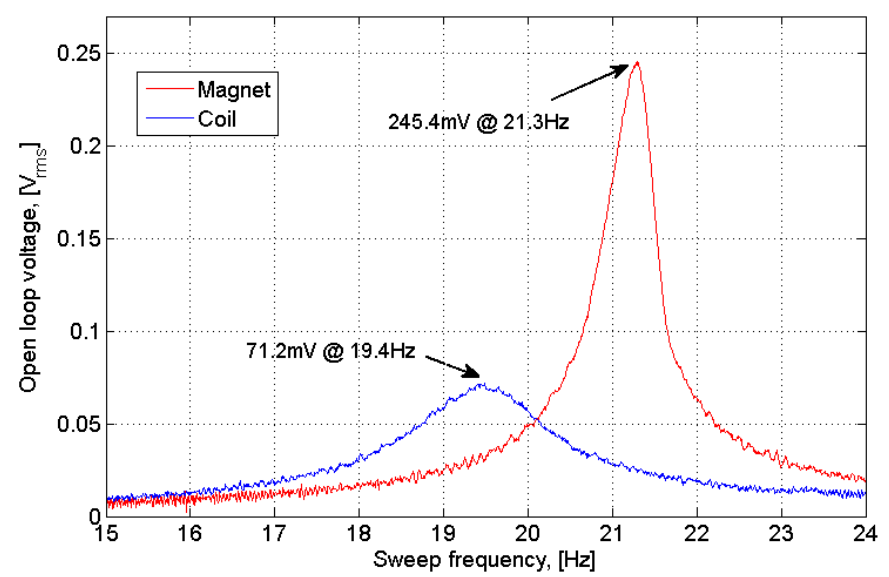

Fig. 18- Frequency response of coil and magnet resonator

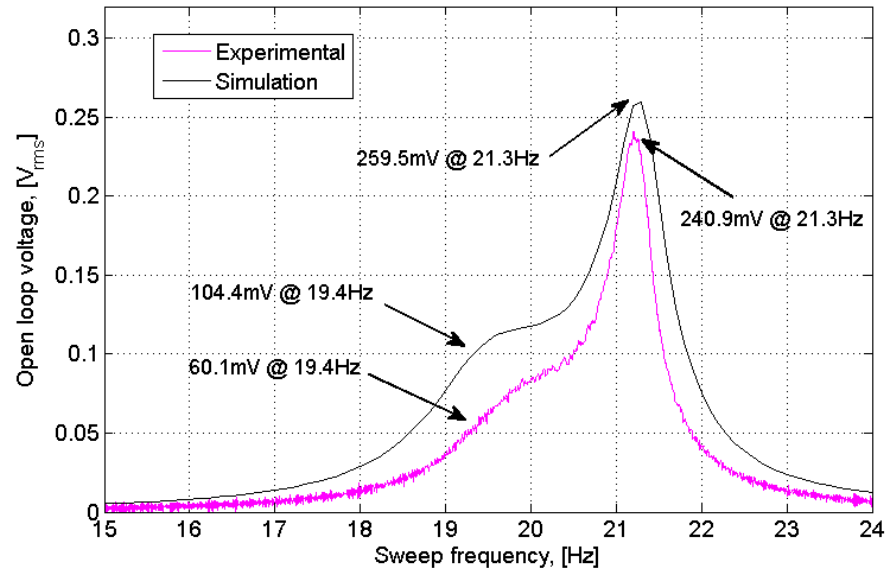

Fig. 19-Frequency response of dual-resonator generator

Lastly, the benchmarking of the output response of a dual-resonator generator versus both coil and magnet resonators (similar to Fig. 18) are shown in Fig. 20. It shows that the dual-resonator generator is able to improve the output response in the Intermediate Region across the range of sweep frequencies. At the intersection point between the coil and magnet plots at $20.1 \mathrm{~Hz}$, the output of the conventional single resonator generator was measured to be $52.9 \mathrm{mV}$. Whereas for the dual-resonator generator, the output was improved and experimentally measured at $83.7 \mathrm{mV}$, which is around $58 . \%$ improvement. However, it may also be noted that improving the Intermediate Region output by using the dual-resonator, has the effect of diminishing the output across the lower $\left(<f_{c}\right)$ and higher $\left(>f_{m}\right)$ frequencies range. This is because when the sweep frequency deviates away from Intermediate Region, the resultant phase angle between the coil and magnet resonators will be relatively low as explained in Fig. 5, hence leading to the lower induced voltage. For the reason of higher damping factor of the coil resonator, the lower frequencies range has a more significant output deterioration as compare to the higher frequencies side. However, it is believed that the deterioration can be improved for the coil resonator by reducing its damping factor [23]. Such as increasing the coil volume, yet its weight or fabricating the coil holder with another highly dense non-magnetic material, such as Tungsten, instead of stainless steel (Density of, Tungsten: $16870 \mathrm{~kg} / \mathrm{m}^{3}$, Stainless steel: $8000 \mathrm{~kg} / \mathrm{m}^{3}$ ). In Fig. 20, it may be also noted that at frequencies around $21.3 \mathrm{~Hz}$, the difference between the peak output response of dual-resonator and magnet devices is very small $(240.9 \mathrm{mV}$ versus $245.4 \mathrm{mV}$ ), or less than $2 \%$ error range. Hence, this further justify that the aforementioned relative induced damping coefficient, $\mathrm{b}_{\mathrm{r}}$, is negligible and it is practically possible to ignore it in the modelling.

For open circuit operation, there is no energy transfer to the electrical domain. To analyze the closed-circuit behavior of the dual-resonator generator, the generator output would need to be measured under electrical loaded conditions. However to date, it has not proven possible to derive a closed form expression for electrical damping factor and the power generated under loaded condition for a dual-resonator generator. Therefore, the power analysis of this generator is set beyond the scope of this paper. The lack of an analytical solution means that there is limited insight into methods which might make it possible to improve the Intermediate Region output without sacrificing the side frequencies (frequencies lying outside the Intermediate Region) output. However, this problem is avoidable if the input frequencies to the generator device always lies within the Intermediate Region. 


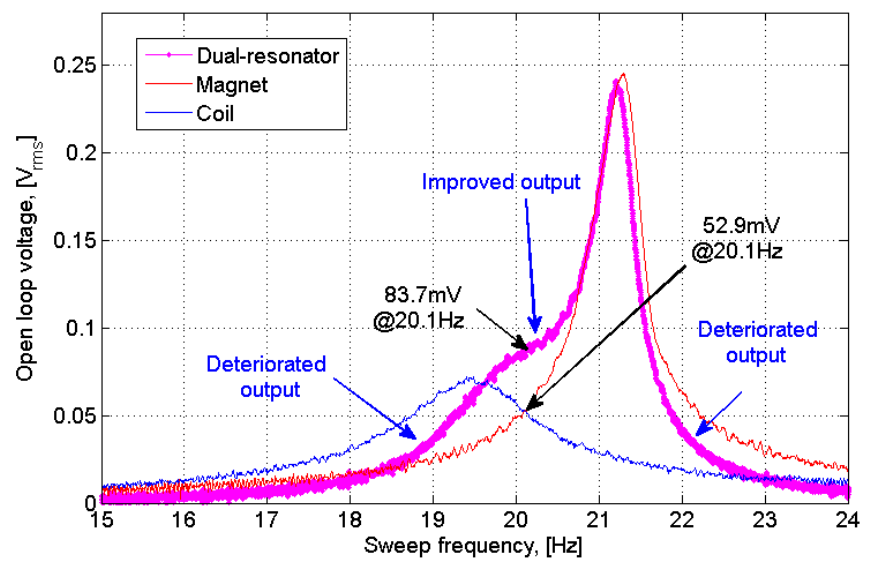

Fig. 20-Benchmarking of output of dual resonator versus coil and magnet resonator

\section{Conclusions and further work}

This paper presented a novel dual-resonator method to improve the bandwidth of a vibration-based generator device. A numerical model of the system is implemented in the Simulink solver. The magnetic circuit and the practical prototype are fabricated based on the results estimated from the ANSYS FEM optimization and material fatigue limit analysis. From a low amplitude vibration source of $0.8 \mathrm{~ms}^{-2}$, the output result has successfully demonstrated that the dual-resonator generator is able to improve the output voltage amplitude in the Intermediate Region, thus giving a wider operational bandwidth for the generator. The maximum output improvement of 58.22\% is recorded at the intersection point between the two output plots from the conventional single resonator generators (moving coil or moving magnet). However, the drawback is that the dual-resonator will produce a lower generated output at the side frequencies. Therefore, the proposed model would be more appropriate for use in applications where the vibration source frequencies vary, but the variation is limited to certain continuous range of the frequency spectrum rather than intermittent across a wide range of the spectrum. Such as behaviour might be encountered in condition monitoring systems for a machine (e.g. pump, vehicle engine etc.) running at certain frequency rate during the particular mode/gear ratio but which might vary within a certain range under different loading conditions. One must also realized that the device could be scaled to other frequencies by redesigning the generator's properties such as beams' dimensions, tip end masses and materials used. Lastly, it is noteworthy to mention that the size of the dual-resonator device is smaller than two separate devices since most of the magnet and coil resonator parts are interleaved and share the common space. Yet, this device beneficial from the higher induced voltage in the Intermediates Region, hence greater bandwidth and output density than a conventional beam-based resonance systems.

Future work will consider how to further reduce the damping factor of the resonators, especially the coil's beam. Also, power analysis, generator structure and concepts to further miniaturize the prototype without loss of conversion efficiency will be examined. Thus future design of the dual-resonator will attempt to improve the Intermediate Region output, yet reduce the deterioration in output at the side frequencies, reduce the size of the generator and increase the electromagnetic coupling for better induced voltage.

\section{Acknowledgments}

The authors would like to thank to Universiti Teknologi PETRONAS for funding this research work under the university internal grant (IGen060/2013). Also the Design \& Prototyping Centre (DPC) for the help in realization of prototype and Department of Mechanical Engineering for the use of the vibration shaker system.

\section{References}

[1] L. Tang, Y. Yang and C.K. Soh, Toward Broadband Vibration-based Energy Harvesting, Journal of Intelligent Material Systems and Structures, Vol 21, Dec 2010, p1867.

[2] R.Elfrink, S.Matova, C. De Nooijer, M. Jambunathan, M. Goedbloed, J. Van de Molengraft, V. Pop, R.J.M. Vullers, M. Renaud and R. Van Schaijk, Shock induced energy harvesting with a MEMS harvester for automotive applications, in Proc. IEDM 2011, Washington DC, USA, Dec. 2011, pp.29.5.1-29.5.4 
[3] L.M.L Oliveira and J.J.P Rodrigues, Wireless Sensor Networks: a Survey on Environmental Monitoring, Journal of Communications, Vol 6, No. 2, Apr. 2011, pp143-151

[4] J. Ko, C. Lu, M.B.Srivastava, J. A Stankovic, A. Terzis and M. Welsh, Wireless Sensor Networks for Healthcare, Proceedings of IEEE, Vol 98, Issue 11, Nov 2010, pp 1947-1960

[5] Z. Zhang, Q. Chen, T. Bergarp, P. Norman, M. Wikstrom, X Yan and L. Zheng, Wireless sensor networks for logistics and retail, Published in Networked Sensing Systems (INSS), 2009 6th International Conference, Pittsburgh, June 2009, page 1-4.

[6] S.M. Diamond and M. G Ceruti, Application of Wireless Sensor Network to Military Information Integration, Industrial Informatics, 5th IEEE International Conference, Vol 1, Vienna, 2007, p317-322

[7] S. Roundy, P.K.Wright and J. Rabaey, A Study of Low Level Vibrations as a Power Source for Wireless Sensor Nodes, Comput. Commun., 26, ,2003,1131-1144

[8] J.M.Gilbert and F Balouchi, Comparison of Energy Harvesting Systems for Wireless Sensor Networks, International Journal of Automation and Computing, vol. 5, 2008, pp334-347

[9] H. A. Sodano, J. Lloyd and D. J. Inman, An Experiment comparison between several active composite actuators for power generation, Smart material Structure, 15, 2005, p799-807

[10] S. Meninger, J.O.Mur-Miranda, R. Amirtharaja, A. Chandrakasan and J.H. Lang, Vibration-to-electric energy conversion, Very Large Scale Integration (VLSI) Systems, IEEE Transactions, 9, 2001, p64-76

[11] Y. Bin, C. Lee, W. Xiang, J. Xie, J.H He, R. K. Kotlanka, S. P. Low and H Feng, Electromagnetic energy harvesting from vibrations of multiple frequencies, Journal of Micromechanics and Microengineering, Vol 19, No. 3, 2009, pp8

[12] C. Peters, D. Maurath, W. Schock, F. Mezger and Y. Manoli, A closed-loop Wide-range Tunable Mechanical Resonator for Energy Harvesting Systems, Journal of Micromechanic and Microengineering, 19, 2009, 094004

[13] M. Wischke, M. Masur, F. Goldschmidtboeing and P. Woias, Electromagnetic Vibration Harvester with Piezoelectrically Tunable Resonance Frequency, Journal of Micromechanic and Microengineering, 20, 2010, 035025

[14] F.Cottone, H. Vocca and L. Gammaitoni, Nonlinear Energy Harvesting, Phys. Rev. Lett., 102, 2008, 080601

[15] O. Zorlu, E.T. Topal and H. Kulah, A Vibration-Based Electromagnetic Energy Harvester Using Mechanical Frequency Up-Conversion Method, IEEE Sensors Journal, vol 11, No 2, Feb 2011, 481-488

[16] K. Ashraf, M.H.Md. Khir, J.O.Dennis and Z.Baharudin, Improved energy harvesting from low frequency vibrations by resonance amplification at multiple frequencies, Sensors and Actuators A, 195, 2013, 123-132

[17] J. Q. Liu, H.B.Fang, Z. Y.Xu, X.H.Mao, X.C.Shen and D.Chen, A MEMS-based piezoelectric power generator array for vibration energy harvesting, Microelectronics Journal, 39, 5, 2007, p802-806

[18] I. Sari, T. Balkan and H. Kulah, An Electromagnetic micro power generator for wideband environmental vibrations, Sensors and Actuators, A145, 2008, 405-413.

[19] C.B.Williams and Y.B.Yates, Analysis of a micro-electric generator for microsystems, Sensors and Actuators A, 52, $1996,8-11$.

[20] B.L. Ooi, Optimisation and Frequency Tuning Concepts for a Vibration Energy Harvester, Ph.D Thesis, 2010, University of Hull, United Kingdom.

[21] A.A. Shabana, Theory of Vibration: An Introduction, second ed., Springer, 1996, New York.

[22] Magnetics: Design Guides, [Online], Available: http://www.mag-inc.com/design/design-guides, 23 Oct , 2013.

[23] B.L.Ooi, A.Rashid.A.Aziz and J.M.Gilbert, Analytical and Numerical modelling for Dual-resonator Vibration-based Electromagnetic Generator, $5^{\text {th }}$ International Conferences on Intelligent \& Advanced System, submitted on $7^{\text {th }}$ Nov 2013, Under Review.

\section{Biographies}

B.L.Ooi obtained a master of engineering degree from the University of Hull, UK, in 2007 and with Ph.D. in energy harvesting in 2011. He is currently a postdoctoral researcher in Centre for Automotive Research \& Electric Mobility (CAREM), Universiti Teknologi PETRONAS, Malaysia. His research interest includes vibration energy harvesting devices for environmental sensing purposes.

A.Rashid.A.Aziz received his Bachelor of Science in 1988, the Master of Science degree in 1990 and a Ph.D. degree in Mechanical Engineering from the University of Miami, USA, in 1995. He is currently a Professor and Head of Centre for Automotive Research and Electric Mobility (CAREM), Universiti Teknologi PETRONAS. From 1998 to 2000 he was the Head of Department of Mechanical Engineering, Universiti Teknologi PETRONAS. His research interests include internal combustion engines, fluid mechanics, computational fluid dynamics, heat transfer and alternative energy. 
J.M.Gilbert graduated from University of Hull, UK, with a bachelor degree in electronic engineering in 1986 and with Ph.D. in robot control in 1989. He has since been a lecturer in the School of Engineering at the same university and served as the Head of Engineering Department from 2011 to 2013. His research interest includes energy harvesting devices, particularly for sensor network applications. 